\title{
CPM Training Waveforms With Autocorrelation Sidelobes Close To Zero
}

\author{
Zilong Liu, Yong Liang Guan, and Chee-Cheon Chui
}

\begin{abstract}
Continuous phase modulation (CPM) plays an important role in wireless communications due to its constant envelope signal property and tight spectrum confinement capability. Although CPM has been studied for many years, CPM training waveforms having autocorrelations with zero sidelobes have not been reported before, to the best of our knowledge. Existing works on CPM system design mostly assume that the channel fading coefficients are either perfectly known at the receiver or estimated using random CPM training waveforms. In this work, we propose a novel class of CPM training waveforms displaying autocorrelation sidelobes close to zero. The key idea of our construction is to apply differential encoding to Golay complementary pair having perfect aperiodic autocorrelation sum properties.
\end{abstract}

Index Terms-Continuous phase modulation, Golay complementary pair (GCP), autocorrelation function, Laurent decomposition, Rimoldi decomposition, differential encoding, channel estimation.

\section{INTRODUCTION}

Continuous phase modulation (CPM) is an attractive nonlinear modulation scheme whose signals exhibit properties of constant envelope and tight spectrum confinement [1]. The first property will allow the transmitter to enjoy high power transmission efficiency as CPM signals have peak-to-average power ratio (PAPR) of 1 (theoretically). Hence, traditional transmission techniques (e.g., [2], [3]) to deal with high PAPR problem in orthogonal frequency-division multiplexing (OFDM) may be avoided when high-rate transmission is not a must. The second property implies less amount of out-of-band power leakage compared to 2- and 4-ary PSK modulations [1], therefore, causing less interference to other applications (operated over adjacent spectral bands) and leading to relatively higher spectral efficiency.

Nowadays, CPM has been used in many areas such as global system for mobile communications (GSM) [4], military and satellite communications [5], millimeter communications [6], and machine-type communications in 5G [7], [8]. In dispersive channels, frequency-domain equalization (FDE) is needed to suppress the effect of intersymbol interference (ISI), followed

Zilong Liu was with the School of Electrical and Electronic Engineering, Nanyang Technological University, Singapore. He is now with Institute of Communication Systems, Home of 5G Innovation Centre, University of Surrey, UK (E-mail: zilong.liuesurrey.ac.uk). Yong Liang Guan is with the School of Electrical and Electronic Engineering, Nanyang Technological University, Singapore (E-mail: eylguanentu.edu.sg). C.C. Chui is with DSO National Laboratories, Singapore 609081 (e-mail: ccheeche@dso.org.sg). The work of Z. Liu and Y. L. Guan was supported by the Advanced Communications Research Program under grant contract DSOCL14095. The work of Z. Liu was also supported in part by National Natural Science Foundation of China under Grant 61750110527, a Research Fund for International Young Scientists. by CPM demodulation as in flat-fading channels. FDE based CPM receiver design can be found in [9]-[11], in which the channel fading coefficients are assumed to be perfectly known at the receiver. Recently, random sequence based channel estimation and equalization has been investigated in [12].

Despite of a long history of CPM research, less has been understood on the training waveform design of CPM. According to [13], the 8 training binary sequences defined in GSM standard [14] have been found by computer search over all possible $2^{16}$ binary sequences. In 2013, Hosseini and Perrins studied the training sequence design of burst-mode CPM over additive white Gaussian noise (AWGN) channels [15], [16]. However, the CPM training sequences proposed in [15] may not be applicable in frequency-selective channels (as will be shown in Section IV). Motivated by this, we target at a systematic construction of CPM training waveforms for frequency-selective channels. Our main idea is to apply differential encoding to Golay complementary pair (GCP) whose aperiodic autocorrelation sums diminish to zero for all the non-zero time-shifts [17]. Taking advantage of Laurent decomposition [18], we show that the resultant CPM training waveform (with modulation index of $h=1 / 2$ ) displays autocorrelation sidelobes close to zero.

\section{PRELIMINARIES}

\section{A. Introduction to $C P M$}

Let $j=\sqrt{-1}$. An equivalent lowpass $M$-ary CPM waveform $s(t)$ is expressed as $s(t)=\exp [j \phi(t ; \mathbf{I})]$, where $\phi(t ; \mathbf{I})=2 \pi h \sum_{k=0}^{n} I_{k} q(t-k T)(n T \leq t \leq(n+1) T)$ is the time-varying phase depending on the information sequence $\mathbf{I}=\left\{I_{k}\right\}_{k=0}^{n}$ with $I_{k}$ being the $k$-th CPM symbol drawn from the set of $\{ \pm 1, \pm 3, \cdots, \pm(M-1)\}, h$ is the modulation index, and $T$ is the symbol duration. The phase-shaping waveform $q(t)$ is defined as the integral of the frequencyshaping pulse $g(t)$ of duration $L T$, i.e., $q(t)=\int_{0}^{t} g(\tau) d \tau$, with $q(t)=0$ for $t \leq 0$ and $q(t)=1 / 2$ for $t \geq L T$. $s(t)$ is called full-response if $L=1$ and partial-response when $L>1$. Note that $\phi(t ; \mathbf{I})$ can be written as

$$
\phi(t ; \mathbf{I})=2 \pi h \sum_{k=0}^{n-L} I_{k} q(t-k T)+2 \pi h \sum_{k=n-L+1}^{n} I_{k} q(t-k T) .
$$

Let $\theta_{n}=\pi h \sum_{k=0}^{n-L} I_{k}$ and $\sigma_{n}=\left[I_{n-1}, I_{n-2}, \cdots, I_{n-L+1}\right]$. It is easy to see that the phase $\phi(t ; \mathbf{I})$ depends on the modulator state $\chi_{n}=\left[\theta_{n}, \sigma_{n}\right]$, where $\theta_{n}$ and $\sigma_{n}$ are called the phase state and the correlative state, respectively. 
Laurent's Decomposition: Laurent showed that a binary partial-response CPM signal can be represented as a superposition of a number of pulse-amplitude-modulated (PAM) pulses [18]. To introduce this, we first define

$$
s_{0}(t)= \begin{cases}\frac{\sin 2 \pi h q(t)}{\sin \pi h}, & 0 \leq t \leq L T, \\ \frac{\sin [\pi h-2 \pi h q(t-L T)]}{\sin \pi h}, & L T \leq t \leq 2 L T, \\ 0, & \text { otherwise }\end{cases}
$$

Also, denote by $a_{p, m} \in\{0,1\}$ the coefficients of the binary representation of integer $p$ in the set of $\left\{0,1, \cdots, 2^{L-1}-1\right\}$, i.e., $p=\sum_{m=1}^{L-1} 2^{m-1} a_{p, m}$. Then, the CPM signal can be written as

$$
s(t)=\sum_{n} \sum_{p=0}^{2^{L-1}-1} \exp \left[j \pi h A_{p, n}\right] \cdot c_{p}(t-n T),
$$

where $c_{p}(t)=s_{0}(t) \prod_{i=1}^{L-1} s_{0}\left[t+\left(i+L a_{p, i}\right) T\right]$, for $0 \leq t \leq$ $T \times \min _{i=1,2, \cdots, L-1}\left[L\left(2-a_{p, i}\right)-i\right]$, and $A_{p, n}=\sum_{m=0}^{n} I_{m}-$ $\sum_{m=1}^{L-1} I_{n-m} a_{p, m}$. In general, $c_{0}(t)$ is the most important PAM $m=1$
pulse as it carries more than $99 \%$ of the total signal energy [18]. Therefore, the CPM signal can be approximated as

$$
s(t) \approx \alpha(t) \triangleq \sum_{n} \gamma_{n} \cdot c_{0}(t-n T)
$$

with

$$
\gamma_{n} \triangleq \exp \left[j \pi h A_{0, n}\right]=\exp \left[j \pi h \cdot \sum_{m=0}^{n} I_{m}\right] .
$$

For ease of presentation, $\left\{\gamma_{n}\right\}$ are called CPM pseudosymbols.

\section{B. Introduction to Golay Complementary Pair (GCP)}

Denote by $\rho_{\mathbf{C}}(k)$ the aperiodic auto-correlation function (AACF) of $\mathbf{C}=\left[C_{0}, C_{1}, \cdots, C_{N-1}\right]$ which is defined as

$$
\rho_{\mathbf{C}}(k)= \begin{cases}\sum_{n=0}^{N-1-k} C_{n} C_{n+k}^{*}, & \text { if } 0 \leq k \leq N-1 ; \\ \rho_{C}^{*}(-k), & \text { if } 1-N \leq k<0 ; \\ 0, & \text { otherwise. }\end{cases}
$$

Let $(\mathbf{C}, \mathbf{D})$ be a pair of sequences with identical length of $N$. $(\mathbf{C}, \mathbf{D})$ is called a GCP [17] if $\rho_{\mathbf{C}}(k)+\rho_{\mathbf{D}}(k)=0$ for any $k \neq 0$. Note that compared to conventional one-dimensional sequences, the two constituent sequences in a GCP work in a cooperative way to ensure that their out-of-phase aperiodic autocorrelations sum to zero.

Let $\phi_{\mathbf{C}}(k)=\sum_{k=0}^{N-1} C_{k} C_{n+k \text { mod } N}^{*}$ be the periodic autocorrelation function (PACF) of $\mathbf{C}$ at time-shift $k$. Clearly, $\phi_{\mathbf{C}}(k)+\phi_{\mathbf{D}}(k)=0$ for any $k \neq 0(\bmod N)$ if $(\mathbf{C}, \mathbf{D})$ is a GCP.

Denote by $\mathbb{Z}_{q}$ the set of integers modulo $q$. For $\underline{x}=$ $\left[x_{1}, x_{2}, \cdots, x_{\nu}\right] \in \mathbb{Z}_{2}^{\nu}$, a generalized Boolean function (GBF) $f(\underline{x})$ (or $f\left(x_{1}, x_{2}, \cdots, x_{\nu}\right)$ ) is defined as a mapping $f:\{0,1\}^{\nu} \rightarrow \mathbb{Z}_{q}$. Let $\left(i_{1}, i_{2}, \cdots, i_{\nu}\right)$ be the binary representation of the integer $i=\sum_{k=1}^{\nu} i_{k} 2^{k-1}$, with $i_{\nu}$ denoting the most significant bit. Given $f(\underline{x})$ (or $f\left(x_{1}, x_{2}, \cdots, x_{\nu}\right)$ ), define $f_{i} \triangleq f\left(i_{1}, i_{2}, \cdots, i_{\nu}\right)$, and

$$
\boldsymbol{f} \triangleq[f(0,0, \cdots, 0), f(1,0, \cdots, 0), \cdots, f(1,1, \cdots, 1)] .
$$

We present the example below to illustrate GBFs defined above. One can find it useful in understanding the GCP construction in Lemma 1 (which is formed by summation of a series of quadratic and linear terms of GBFs).

Example 1: Let $\nu=3$ and $q=2$. The associated sequences of $1, x_{1}, x_{3}, x_{1} x_{3}$ are

$$
\begin{array}{cc}
\mathbf{1} & =(1,1,1,1,1,1,1,1), \\
\mathbf{x}_{1} & =(0,1,0,1,0,1,0,1), \\
\mathbf{x}_{3} & =(0,0,0,0,1,1,1,1), \\
\mathbf{x}_{1} \mathbf{x}_{3}+\mathbf{1} & =(1,1,1,1,1,0,1,0),
\end{array}
$$

respectively.

Lemma 1: (Davis-Jedwab Construction of GCP [20]) Let

$$
f(\underline{x}) \triangleq \frac{q}{2} \sum_{k=1}^{\nu-1} x_{\pi(k)} x_{\pi(k+1)}+\sum_{k=1}^{\nu} c_{k} x_{k}+c,
$$

where $\pi$ is a permutation of the set $\{1,2, \cdots, \nu\}$, and $c_{k}, c \in$ $\mathbb{Z}_{q}$ ( $q$ even integer). Then, for any $c^{\prime} \in \mathbb{Z}_{q}, \boldsymbol{f}$ and $\boldsymbol{f}+\frac{q}{2} \boldsymbol{x}_{\pi(1)}+$ $c^{\prime} \cdot \mathbf{1}$ form a GCP over $\mathbb{Z}_{q}$ of length $2^{\nu}$.

\section{Proposed CPM Training Waveform Design}

In this section, we will propose a training waveform design for CPM signal $s(t)$ [or the approximation $\alpha(t)$ ] with periodic autocorrelation sidelobes close to zero. Throughout the proposed design, we consider binary CPM with $h=1 / 2$. Therefore, $\exp [j \pi h]=j$. To get started, we first consider a sequence of $\left\{\gamma_{n}\right\}_{n=0}^{2 N-1}$ with $2 N$ non-zero elements satisfying $N>L+1$ and

$$
\gamma_{n}=\gamma_{n+N}, \text { for } 0 \leq n \leq N-1 .
$$

The PACF of the CPM approximation $\alpha(t)$ [cf. (4)] over $[N T, 2 N T]$ is defined as

$$
\phi_{\alpha}(\tau)=\int_{t=N T}^{2 N T} \alpha(t) \alpha^{*}(t+\tau) d t,
$$

where

$$
\alpha(t+\tau)= \begin{cases}\alpha(t+\tau-N T), & \text { if } t+\tau>2 N T ; \\ \alpha(t+\tau+N T), & \text { if } t+\tau<N T .\end{cases}
$$

In addition, the AACF of $c_{0}(t)$, the most significant pulse in Laurent's decomposition, is defined as

$$
\rho_{c_{0}}(\tau)=\int_{t=-\infty}^{+\infty} c_{0}(t) c_{0}(t+\tau) d t,
$$

where $c_{0}(t)=0$ if $t<0$ or $t>(L+1) T$. Hence, $\rho_{c_{0}}(\tau)=0$ if $|\tau|>(L+1) T$. By [19, (6.45b)], we have

$$
\phi_{\alpha}(\tau)=\sum_{k=-\infty}^{+\infty} \phi_{\gamma}(k) \rho_{c_{0}}(\tau-k T)
$$

(12) implies that the PACF of $\alpha(t)$ [i.e., $\rho_{\alpha}(\tau)$ ] is zero for any $|\tau| \geq(L+1) T$ if

$$
\phi_{\gamma}(k)=0, \quad \forall k \neq 0 .
$$




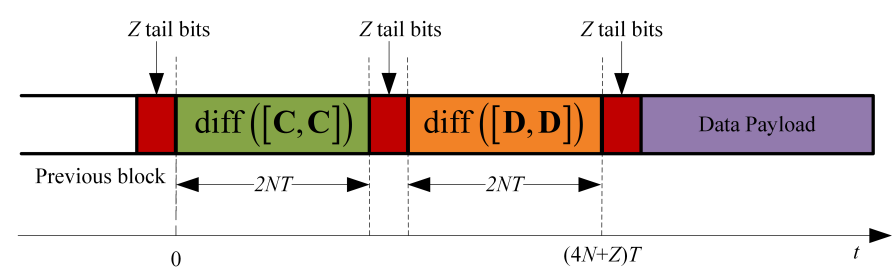

Fig. 1: CPM block structure consisting of training sequence and data payload.

Nevertheless, it is hard to find sequence $\left\{\gamma_{n}\right\}$ satisfying (5) and (13) simultaneously. Because of this, we consider sequence pair $\mathbf{C}=\left[C_{0}, C_{1}, \cdots, C_{N-1}\right]$ and $\mathbf{D}=$ $\left[D_{0}, D_{1}, \cdots, D_{N-1}\right]$ over $\{0,1\}^{N}$. Applying the "differentialencoding", denoted by $\operatorname{diff}(\cdot)$, to $\widetilde{\mathbf{C}} \triangleq[\mathbf{C}, \mathbf{C}]$, we obtain $I_{\mathbf{C}} \triangleq \operatorname{diff}([\mathbf{C}, \mathbf{C}])=\left[I_{C, 0}, I_{C, 1}, \cdots, I_{C, 2 N-1}\right]$ with

$$
\begin{aligned}
I_{C, m} & =\left(2 \tilde{C}_{m}-1\right) \cdot\left(2 \tilde{C}_{m-1}-1\right) \\
& =4 \tilde{C}_{m} \tilde{C}_{m-1}-2 \tilde{C}_{m}-2 \tilde{C}_{m-1}+1 \in\{-1,1\},
\end{aligned}
$$

where $0 \leq m \leq 2 N-1$ and $\tilde{C}_{-1}=1$. It is clear that

$$
\exp \left[\pi h I_{C, m}\right]=j \cdot(-1)^{\tilde{C}_{m}} \cdot(-1)^{\tilde{C}_{m-1}} .
$$

Similarly, applying "differential-encoding" to $\tilde{\mathbf{D}} \triangleq[\mathbf{D}, \mathbf{D}]$, we obtain $I_{\mathbf{D}} \triangleq \operatorname{diff}([\mathbf{D}, \mathbf{D}])=\left[I_{D, 0}, I_{D, 1}, \cdots, I_{D, 2 N-1}\right]$ assuming $\tilde{D}_{-1}=1$. As we will see later, differential-encoding helps to mitigate the correlations among CPM symbols which facilitates the design of CPM training waveforms with autocorrelation sidelobes close to zero. Then, send $I_{\mathbf{C}}$ and $I_{\mathbf{C}}$ for CPM modulation following the transmission structure shown in Fig. 1. The " $Z$ tail bits", placed before $I_{\mathbf{C}}$ (or $I_{\mathbf{D}}$ ), are used to return the CPM modulator phase state to zero. Systematic method for the generation of tail bits can be found in [9] using Rimoldi decomposition [21].

Applying $I_{\mathbf{C}}$ as a sequence of $2 N$ CPM symbols, the CPM pseudo-symbols can be expressed as follows.

1) If $n \in\{0,1,2, \cdots, N-1\}$, we have

$$
\begin{aligned}
\gamma_{C, n} & =\exp \left[j \pi h \cdot \sum_{m=0}^{n} I_{C, m}\right] \\
& =j^{n+1} \cdot(-1)^{C_{n}} \cdot(-1)^{C_{-1}}=j^{n+3} \cdot(-1)^{C_{n}} .
\end{aligned}
$$

2) If $n \in\{N, N+1, N+2, \cdots, 2 N-1\}$, we have

$$
\begin{aligned}
\gamma_{C, n} & =\exp \left[j \pi h \cdot \sum_{m=0}^{n} I_{C, m}\right] \\
& =j^{n-N+3} \cdot(-1)^{C_{n-N}} \cdot j^{N} .
\end{aligned}
$$

When $N \equiv 0 \bmod 4$, one can see that

$$
\left[\gamma_{C, 0}, \gamma_{C, 1}, \cdots, \gamma_{C, N-1}\right]=\left[\gamma_{C, N}, \gamma_{C, N+1}, \cdots, \gamma_{C, 2 N-1}\right]
$$

In this case, (8) holds. This implies that $I_{\mathbf{C}}$ is a periodic transmission of two identical length- $N$ sequences, which in turn allows the calculation of PACF at the local receiver. The approximated CPM waveform $\alpha_{\mathbf{C}}(t)$ in (4) can be written as

$$
\alpha_{\mathbf{C}}(t)=\sum_{n=0}^{2 N-1} \underbrace{j^{n+3} \cdot(-1)^{C_{n}}}_{\gamma_{C, n}} \cdot c_{0}(t-n T) \text {, for } 0 \leq t \leq 2 N T \text {. }
$$

Denote by $\alpha_{\mathbf{D}}(t)$ the approximated CPM waveform (after "differential encoding") corresponding to $I_{\mathbf{D}}$. Similar to (18), we have

$$
\left[\gamma_{D, 0}, \gamma_{D, 1}, \cdots, \gamma_{D, N-1}\right]=\left[\gamma_{D, N}, \gamma_{D, N+1}, \cdots, \gamma_{D, 2 N-1}\right]
$$

for $N \equiv 0 \bmod 4$. Also, similar to (19), we obtain

$$
\alpha_{\mathbf{D}}(t)=\sum_{n=0}^{2 N-1} \underbrace{j^{n+3} \cdot(-1)^{D_{n}}}_{\gamma_{D, n}} \cdot c_{0}(t-n T-2 N T-Z T),
$$

for $2 N T+Z T \leq t \leq 4 N T+Z T$.

Next, we will show $\gamma_{\mathbf{C}} \triangleq\left\{\gamma_{C, n}\right\}_{n=0}^{N-1}$ and $\gamma_{\mathbf{D}} \triangleq\left\{\gamma_{D, n}\right\}_{n=0}^{N-1}$ form a quaternary GCP provided that $(\mathbf{C}, \mathbf{D})$ is a binary GCP generated by the Davis-Jedwab construction (see Lemma 1). In the context of Lemma 1, let

$$
f=\sum_{k=1}^{\nu-1} x_{\pi(k)} x_{\pi(k+1)}+\sum_{k=1}^{\nu} c_{k} x_{k}+c(\bmod 2)
$$

and $f+x_{\pi(1)}+c^{\prime}(\bmod 2)$ be the GBFs of $\mathbf{C}$ and $\mathbf{D}$, respectively, where $N=2^{\nu}(\nu \geq 2$ as $N$ should be divisible by 4 ). Lifting these two GBFs from $\mathbb{Z}_{2}$ to $\mathbb{Z}_{4}$ and noting that $n=\sum_{k=1}^{\nu} x_{k} 2^{k-1}$, the corresponding GBFs of $\gamma_{C, n}$ and $\gamma_{D, n}$ can be expressed as

$$
\begin{aligned}
f_{\mathbf{C}}= & 2 \sum_{k=1}^{\nu-1} x_{\pi(k)} x_{\pi(k+1)}+2 \sum_{k=1}^{\nu} c_{k} x_{k} \\
& +2 c+\sum_{k=1}^{\nu} x_{k} 2^{k-1}+3(\bmod 4) \\
= & 2 \sum_{k=1}^{\nu-1} x_{\pi(k)} x_{\pi(k+1)}+2 \sum_{k=1}^{\nu} c_{k} x_{k} \\
& +2 c+2 x_{2}+x_{1}+3(\bmod 4) \\
= & 2 \sum_{k=1}^{\nu-1} x_{\pi(k)} x_{\pi(k+1)}+\sum_{k=3}^{\nu}\left(2 c_{k}\right) x_{k} \\
& +\left(2+2 c_{2}\right) x_{2}+\left(1+2 c_{1}\right) x_{1}+2 c+3(\bmod 4)
\end{aligned}
$$

and $f_{\mathbf{D}}=f_{\mathbf{C}}+2 x_{\pi(1)}+2 c^{\prime}(\bmod 4)$, respectively. It is clear that $f_{\mathbf{C}}, f_{\mathbf{D}}$ satisfy the GBF forms in Lemma 1 . Thus, $\gamma_{\mathbf{C}}$ and $\gamma_{\mathbf{D}}$ are a quaternary GCP. Applying (12) to (19) and (21), we assert that

$$
\begin{aligned}
& \phi_{\alpha_{\mathbf{C}}}(\tau)+\phi_{\alpha_{\mathbf{D}}}(\tau) \\
= & \sum_{k=-\infty}^{+\infty}\left[\phi_{\gamma_{\mathbf{C}}}(k)+\phi_{\gamma_{\mathbf{D}}}(k)\right] \rho_{c_{0}}(\tau-k T) \\
= & \begin{cases}2 N T \rho_{c_{0}}(\tau), & \text { if } 0 \leq|\tau| \leq(L+1) T ; \\
0, & \text { if }(L+1) T<|\tau| \leq(N-L-1) T .\end{cases}
\end{aligned}
$$


Consider the CPM training waveform over $\{s(t): 0 \leq t \leq$ $(4 N+Z) T\}$ and let

$$
\begin{aligned}
& S_{\mathbf{C}}^{(1)}=\{s(t): 0 \leq t \leq N T\}, \\
& S_{\mathbf{C}}^{(2)}=\{s(t): N T \leq t \leq 2 N T\}, \\
& S_{\mathbf{D}}^{(1)}=\{s(t):(2 N+Z) T \leq t \leq(3 N+Z) T\}, \\
& S_{\mathbf{D}}^{(2)}=\{s(t):(3 N+Z) T \leq t \leq(4 N+Z) T\} .
\end{aligned}
$$

At the receiver, $S_{\mathbf{C}}^{(2)}$ and $S_{\mathbf{D}}^{(2)}$ are taken as two local reference waveforms for correlation with $S_{\mathbf{C}}^{(1)}$ and $S_{D}^{(1)}$, respectively. This is because $S_{\mathbf{C}}^{(1)}$ (and $S_{\mathbf{D}}^{(1)}$ ) will be spread into the time window of $N T \leq t \leq 2 N T$ [and $(3 N+Z) T \leq t \leq(4 N+$ $Z) T$ ] owing to the multipath propagation. Finally, we have the following assertion.

$$
\begin{aligned}
& \phi_{S_{\mathbf{C}}^{(1)}, S_{\mathbf{C}}^{(2)}}(\tau)+\phi_{S_{\mathbf{D}}^{(1)}, S_{\mathbf{D}}^{(2)}}(\tau) \\
\approx & \phi_{\alpha_{\mathbf{C}}}(\tau)+\phi_{\alpha_{\mathbf{D}}}(\tau) \\
\approx & 0, \text { if }(L+1) T<|\tau| \leq(N-L-1) T .
\end{aligned}
$$

\section{Simulation Results}

In the context of Lemma 1, let $q=2, \nu=4$ (i.e., $N=2^{\nu}=$ 16) and $c=0, c^{\prime}=1$. Consider two GCPs, with $\pi=[1,2,3,4]$ and $\left[c_{1}, c_{2}, c_{3}, c_{4}\right]=[1,0,1,1]$ for GCP 1 , and $\pi=[2,3,4,1]$ and $\left[c_{1}, c_{2}, c_{3}, c_{4}\right]=[1,1,0,1]$ for GCP 2 . The resultant GCPs are given below.

$$
\begin{aligned}
& \operatorname{GCP} 1=\left[\begin{array}{l}
+-++-+++-+---+++ \\
---+++-++++-++-+
\end{array}\right], \\
& \operatorname{GCP} 2=\left[\begin{array}{l}
+--+-+-+++--++++ \\
-+-++--+------++
\end{array}\right] .
\end{aligned}
$$

Applying differential encoding to GCPs 1 and 2, we obtain differentially-encoded pairs, denoted by "Diff-GCP 1" and "Diff-GCP 2", respectively. Each pair will be sent as $I_{\mathbf{C}}$ and $I_{\mathbf{D}}$ (see Section III) for CPM modulation following the transmission structure in Fig. 1. For comparison, differential encoding is also applied to GSM sequence $[+-+++---$ $-+---+--]$ [14]. The resultant sequence is referred to as "Diff-GSM" and will be sent as $I_{\mathbf{C}}$ only for CPM training. For simulation, we consider the GMSK frequency pulse $g(t)$ below.

$$
g(t)=\frac{1}{2 T}\left[Q\left(\frac{\frac{t}{T}+\frac{1}{2}}{\sigma}\right)-Q\left(\frac{\frac{t}{T}-\frac{1}{2}}{\sigma}\right)\right],
$$

where $Q(t)=\int_{t}^{+\infty} \frac{1}{\sqrt{2 \pi}} \exp \left(-\frac{x^{2}}{2}\right) d x$ and $\sigma^{2}=\frac{\operatorname{In} 2}{4 \pi^{2}(B T)^{2}}$. Considering GMSK frequency pulse with $B T=0.3$ and truncated frequency pulse length of $L=3$, we obtain CPM training waveform $\{s(t): 0 \leq t \leq(4 N+Z) T\}$, where $Z=3$ in this example. The (normalized) autocorrelation magnitudes of CPM waveforms, i.e., $\left|\phi_{S_{\mathbf{C}}^{(1)}, S_{\mathrm{C}}^{(2)}}(\tau)+\phi_{S_{\mathbf{D}}^{(1)}, S_{\mathbf{D}}^{(2)}}(\tau)\right|$, are shown in Fig. 2. One can see that the proposed training waveforms 1 and 2 (corresponding to Diff-GCPs 1 and 2, respectively) exhibit autocorrelation sidelobes close to zero for time-shifts larger than $(L+1) T$. In contrast, the training waveform from the Diff-GSM sequence exhibits considerably large autocorrelation sidelobes when time-shift is larger than $6 T$. To show the effectiveness of differential-encoding, we also

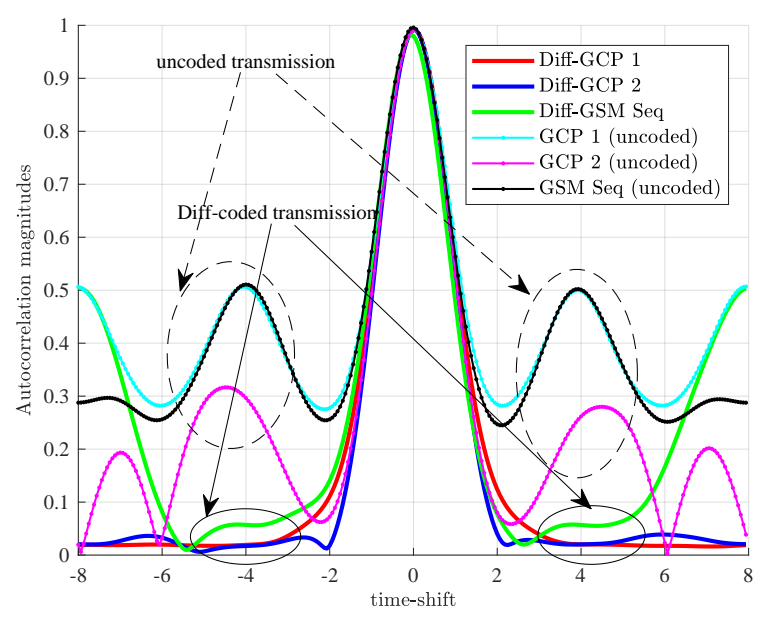

Fig. 2: Autocorrelation comparison of different CPM waveforms

depict the autocorrelation magnitudes of uncoded GCPs 1 and 2 , and uncoded GSM sequence. It is shown that CPM waveforms after differential-encoding exhibit lower autocorrelation sidelobes compared to the uncoded ones.

Next, we apply a set of CPM training waveforms, which are normalized to have identical energy in the transmission, for estimation of a 16-path channel (separated by integer symbols duration) having uniform power delay profile. Specifically, we consider $h[t]=\sum_{n=0}^{15} h_{i} \delta[t-n T]$, where $h_{i}$ 's are complex-valued Gaussian random variables with zero mean and $\mathbb{E}\left(\left|h_{i}\right|^{2}\right)=1 / 16$. These CPM training waveforms are generated based on Diff-GCPs 1 and 2, Diff-GSM, uncoded random sequences, "Diff-Rand" sequences (i.e., differentiallyencoded random "on-the-fly" sequences), uncoded HosseiniPerrins (HP) sequence $[----++++++++----]$ [15], and "Diff-HP" sequence. Using least squares (LS) estimator, comparison of channel estimation mean-squared-errors (MSEs) of different CPM training waveforms is shown in Fig. 3. The CPM training waveforms from uncoded random sequences, Diff-Rand sequences, uncoded HP sequence, and Diff-HP sequence result in relatively higher MSEs due to their autocorrelation sidelobes with larger variations and rankdeficient (sometimes) LS estimator. The GSM training waveform leads to MSE performance $8.5 \mathrm{~dB}$ away from the CramérRao lower bound (CRLB) ${ }^{1}$. The MSEs of using the proposed training waveforms (based on differentially encoded GCPs) exhibit MSEs much closer to CRLB, as close as $4 \mathrm{~dB}$ from the CRLB for the case of "Diff-GCP 1" (compared to 5dB distance for "Diff-GCP 2"). This is understandable as the training waveform from Diff-GCP 1 displays the best autocorrelation performance with uniformly low sidelobes, as shown in Figure 2. It should be noted that the estimates of $h_{1}$ and $h_{15}$ are more likely to suffer from larger MSEs due to the roll-off autocorrelation sidelobes at $\pm T$ (see Figure 2).

Furthermore, under the same frequency-selective channel model, Fig. 4 compares the uncoded bit-error-rates (BERs) of

\footnotetext{
${ }^{1}$ This CRLB is derived for perfect CPM training waveform with zero autocorrelation sidelobes for all the non-zero time-shifts [22].
} 


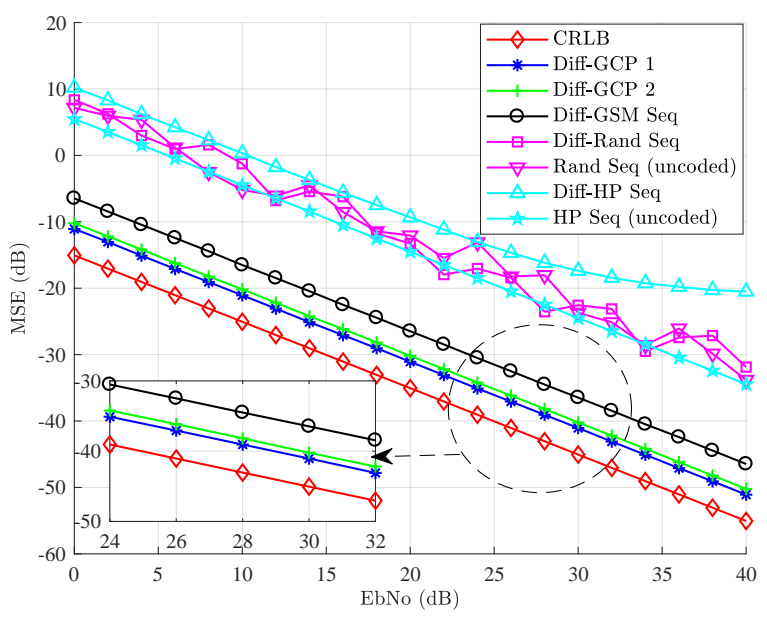

Fig. 3: Comparison of channel estimation MSEs using different CPM training waveforms

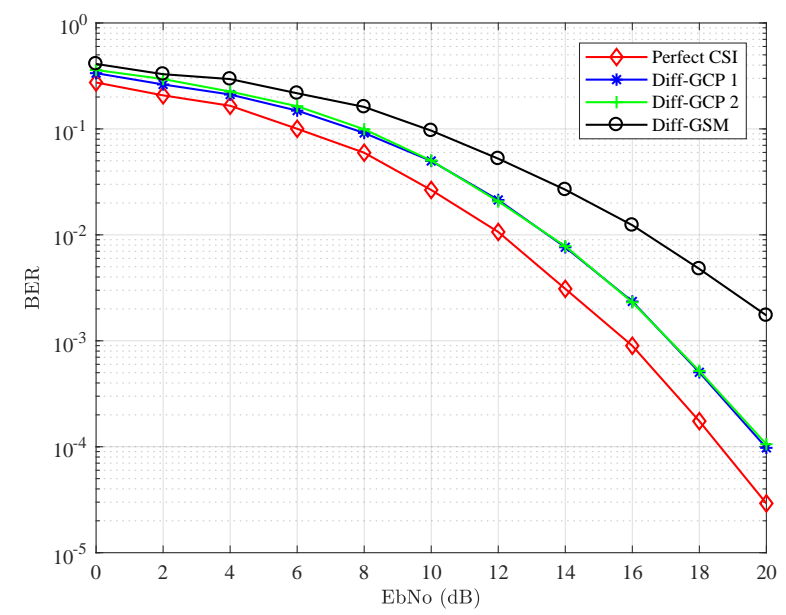

Fig. 4: Comparison of BERs (without error-correcting codes) using different CPM training waveforms

GMSK systems using perfect channel state information (CSI) and estimated CSI from the proposed CPM training waveforms. Here, we follow the CPM receiver design developed in [9], where single-carrier frequency-domain equalization (SC-FDE) with minimum MSE is adopted. It is seen that the BER curves corresponding to the two Diff-GCPs are very close, and are about $1.4 \mathrm{~dB}$ to the BER curve with perfect CSI. On the other hand, the BER curve corresponding to Diff-GSM displays $5 \mathrm{~dB}$ distance to that with perfect CSI.

\section{CONCLUSIONS}

A systematic construction of CPM training waveform displaying autocorrelation sidelobes close to zero has been proposed. Our idea is to apply differential-encoding to a GCP and then send the encoded component sequences one after another, separated by tail bits, to the CPM modulator. Note that this work is focused on binary CPM with modulation index of $1 / 2$. It would be interesting to extend the presented training waveform design to generic CPM schemes (e.g., non-binary modulation orders, rational/integer modulation indices) using CPM decompositions reported in [23] and [24].

\section{REFERENCES}

[1] J. B. Anderson, T. Aulin, and C. E. Sundberg, Digital Phase Modulation, New York: Plenum, 1986.

[2] T. Jiang and G. Zhu, "Complement block coding for reduction in peakto-average power ratio of OFDM signals," IEEE Communi. Mag., vol. 43, no. 9, S17-S22, Sept. 2005.

[3] T. Jiang and Y. Wu, "An Overview: Peak-to-Average Power Ratio Reduction Techniques for OFDM Signals," IEEE Trans. Broadcast., vol. 54, no. 2, pp. 257-268, Jun. 2008.

[4] Digital Cellular Telecommunications System, Modulation (GSM 05.04), ETSI standard ETS 300959, pp. 1-10, May. 1997.

[5] A. Emmanuele, A. Zanier, F. Boccolini, et al., "Spread-spectrum continuous-phase-modulated signals for satellite navigation," IEEE Trans. Aeros. Electro. Systems, vol. 48, no. 4, pp. 3234-3249, Oct. 2012.

[6] R. C. Daniels and R.W. Heath, Jr., " $60 \mathrm{GHz}$ wireless communications: emerging requirements and design recommendations," IEEE Veh. Technol. Mag., vol. 2, no. 3, pp. 41-50, Sept. 2007.

[7] M. Wylie-Green, E. Perrins and T. Svensson, "Introduction to CPMSCFDMA - a novel multiple-access power-efficient transmission scheme," IEEE Trans. Commun., vol. 7, no. 59, pp. 1904-1915, Jul. 2011.

[8] C. Bockelmann et al, "Massive machine-type communications in 5G: physical and MAC-layer solutions," IEEE Commun. Magazine, pp. 5965, Sep. 2016.

[9] J. Tan and G. L. Stuber, "Frequency-domain equalization for continuous phase modulation," IEEE Trans. Wireless Commun., vol. 4, pp. 2479-2490, 2005.

[10] F. Pancaldi and G. M. Vitetta, "Equalization algorithms in the frequency domain for continuous phase modulations," IEEE Trans. Commun., vol. 54 , no. 4, pp. 648-658, 2006.

[11] W. Van Thillo, F. Horlin, J. Nsenga, V. Ramon, A. Bourdoux, and R. Lauwereins, "Low-complexity linear frequency domain equalization for continuous phase modulation," IEEE Trans. Wireless Commun., vol. 8 , no. 3, pp. 1435-1445, 2009.

[12] R. Chayot, N. Thomas, C. Poulliat, M.-L. Boucheret, N. V. Wambeke and G. Lesthievent, "Channel estimation and equalization for CPM with appliation for aeronautical communications via a satellite link," in IEEE Military Communications Conference (MILCOM), Baltimore, MD, pp. 888-893, Oct. 2017.

[13] M. Drutarovsky, "GSM channel equalization algorithm - modern DSP coprocessor approach," Radioengineering, vol. 8, no. 4, pp. 26-31, Dec. 1999.

[14] Digital cellular telecommunications system (Phase 2), Multiplexing and multiple access on the radio path (GSM 05.02), ETSI standard ETS 300574, pp. 1-39, Apr. 1997.

[15] E. Hosseini and E. Perrins, "The Cramér-Rao bound for training sequence design for burst-mode CPM," IEEE Trans. Commun., vol. 61, no. 6, pp. 2396-2407, Jun. 2013.

[16] E. Hosseini and E. Perrins, "Timing, carrier, and frame synchronization of burst-mode CPM," IEEE Trans. Commun., vol. 61, no. 12, pp. 51255138, Dec. 2013

[17] M. J. E. Golay, "Complementary series," IRE Trans. Inf. Theory, vol. IT-7, pp. 82-87, Apr. 1961.

[18] P. A. Laurent, "Exact and approximate construction of digital phase modulations by superposition of amplitude modulated pulses," IEEE Trans. Commun., vol. COM-34, no. 2, pp. 150-160, Feb. 1986.

[19] J. S. Lee and L. E. Miller, CDMA Systems Engineering Handbook, Mobile Communications series, Artech House Publishers, 1998.

[20] J. A. Davis and J. Jedwab, "Peak-to-mean power control in OFDM, Golay complementary sequences, and Reed-Muller codes," IEEE Trans. Inf. Theory, vol. 45, pp. 2397-2417, Nov. 1999.

[21] B. E. Rimoldi, "A decomposition approach to CPM," IEEE Trans. Inf. Theory, vol. 34, no. 2, pp. 260-270, Mar. 1988.

[22] A. Milewski, "Periodic sequences with optimal properties for channel estimation and fast start-up equalziation," IBM J. Res. Develop., vol. 27, no. 5, pp. 426-439, 1983.

[23] U. Mengali and M. Morelli. "Decomposition of M-ary CPM signals into PAM waveforms," IEEE Trans. Inf. Theory, vol. 41, no. 5, pp. 1265-1275, Sept. 1995.

[24] X. Huang and Y. Li, "The PAM decomposition of CPM signals with integer modulation index," IEEE Trans. Communi., vol. 51, no. 4, pp. 543-546, Apr. 2003. 